\title{
Capabilities of smart antenna in tracking the desired signal in wireless communication system through non-blind adaptive algorithms
}

\author{
Edvin J. Kitindi \\ Department of Informatics, Faculty of Science, Sokoine University of Agriculture, Morogoro, Tanzania
}

\begin{abstract}
Future wireless systems will require higher data rates with better coverage for a wide variety of users operating with a large variety of different systems. To achieve these goals, greater power, interference suppression, and multipath mitigation are needed. Smart antenna gives a promising future for wireless communication systems since it can improve the capacity, extend coverage and provide better quality services by tracking users, switching beams accordingly. In this paper three non-blind adaptive beamforming algorithms, least mean square (LMS), Recursive Least square (RLS) and Sample matrix inversion (SMI) have been studied. Investigation of their performances in tracking a desired user has been made and compared. All the three algorithms direct their beams towards a desired user and place nulls towards interferer. However the rate of their performance differs from each other.
\end{abstract}

Keywords: Non-blind adaptive algorithms, beamforming, LMS, RLS, SMI, Desired user, desired signal

\section{INTRODUCTION}

The explosive growth of the number of digital cellular subscribers, many service providers increasingly are concerned with the limited capacities of their existing networks [1]. This concern has led to the deployment of smart antenna systems in the cellular network systems. The smart antenna system typically employs multibeam technologies, being shown through extensive analysis, simulation, and experimentation, to provide substantial performance improvements in FDMA, TDMA and CDMA networks [2-6].

However multipath and co-channel interference are among the characteristics that limits the performance and capacity in mobile communication systems [7]. Smart antenna is among the promising technologies that can raise the capacity in wireless networks by effectively reducing multipath and co-channel interference [7-10]. Smart antennas employ a set of radiating elements arranged in the form of array. The signals from these elements are combined to form a movable or switchable beam pattern that follows the desired user
The process of combining the signals and then focusing the radiation in a particular direction is often referred to as digital beamforming [8], [9].

A Smart Antenna System [11], [12] can suppress interferences, provide higher system capacity, improves quality of service and higher frequency reuse, and reduces power consumption. From a practical point of view, a Smart Antenna System combines an antenna array with digital signal processing techniques (adaptive beamforming techniques, direction of arrival procedures, etc.) in order to obtain a software steerable antenna pattern and direct the radiated power in (or receive from) the desired direction only [14], [15]. Therefore a smart antenna is defined as an array of antennas with a digital signal processing unit that can change its pattern dynamically towards a desired users and place nulls to the direction of noise, interference and multipath signals. Figure 1 shows the general structure of the smart antenna.

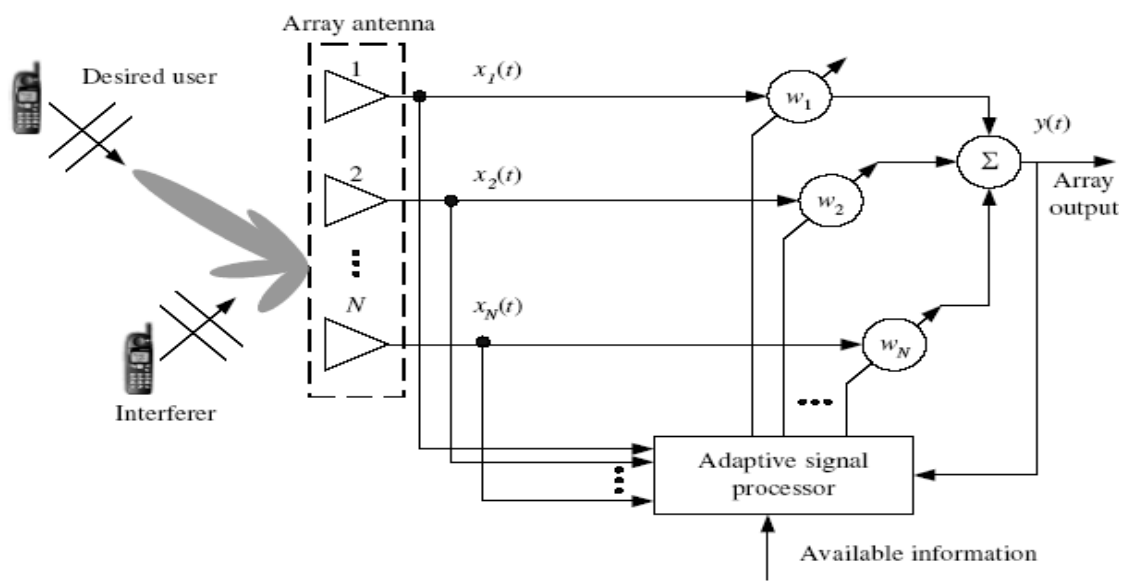

Figure 1: Structure of a smart antenna system [12]. 
The early smart antenna systems were designed for use in military applications to suppress interfering or jamming signals from the enemy [13]. Since interference suppression was a feature in this system, this technology was borrowed to apply into personal wireless communications where interference was limiting the number of users that a network could handle. It was a major challenge to apply smart antenna technology to personal wireless communications since the traffic is denser. Also, the time available for complex computations was limited. However, the advent of powerful, low-cost, digital processing components and the development of software-based techniques have made smart antenna systems a practical reality for cellular communications systems.

The high data rate requirements for wireless services grows every day but the radio frequency (RF) i.e spectrum remains the same, this brings a big challenge in the field. However it have been noticed that most of this resource is underutilized and therefore there is a need to establish a method that will allow the spectrum sharing. The use of adaptive antenna array is one of the approaches that show real promise for effective spectrum utilization. Its availability is of vital importance, as it gives the ability for the transmitter to exploit the geographic awareness and focus its radiation patterns towards the direction of the receiver, whereby at the receiver, beamforming is performed for the adaptive cancellation of residual interference from known transmitters, as well as interference produced by other unknown transmitters.

In a Smart antenna system the arrays by themselves are not smart, it is the digital signal processing that makes them smart. But it should be noted that the term "smart" refers to the whole antenna system and not just the array antenna alone [12]. That is the availability of the adaptive beamforming algorithm makes the antenna to be smart. Therefore in this study we explore the performance of non-blind adaptive beamforming algorithms on smart antenna in tracking the desired user in wireless communication systems.

\section{NON-BLIND ADAPTIVE BEAMFORMING} ALGORITHM

In this paper the tracking of the desired signal of the least mean square (LMS), Recursive Least square (RLS) and Sample matrix inversion (SMI) in SA have been studied. The performance comparison of these algorithms is based on the amplitude enhanced towards the desired user as it has been shown in the simulations.

\section{1) A. Least Mean Square (LMS) Algorithm}

The Least mean square algorithm is a gradient based quadratic approach. It estimates the gradient vector from the available data and updates the weight vector in accordance with the incoming data [16], [17]. It uses the estimated gradient vector due to the fact that it is impossible to obtain exactly gradient vector for each iteration; hence difficult to choose a suitable step-size parameter that make it difficult in reaching the optimum weight vector. This algorithm intends to minimize the Mean square error (MSE). The best way to establish the minimum is through gradient method in which the cost function is to find the MSE.

Figure 2 show the LMS beamforming network in linear array of SA whereby the signal $x(t)$ received by multiple antenna elements is multiplied with the coefficients in a weight vector $w$ (a series of amplitude and phase coefficients) which adjust the phase and the amplitude of the incoming signal accordingly. $y(t)$ is the result of the summed weight signal received by the antenna elements. An adaptive algorithm is then employed to minimize the error $e(t)$ between a desired signal $d(t)$ and the array output $y(t)$ given by linear combination of the data at the $N$ SA sensors/elements.

The LMS can be summarized in the following functions:

Array output: $\quad y(k)=w^{H}(k) x(k)$

Error:e $(k)=d(k)-y(k)$

Weight vector updates: $w(k+1)=w(k)+$

$\mu x(k) e^{*}(k)$

The response of LMS algorithm is determined by three principal factors step size parameter $\mu$ number of weights and eigen-value of the correlation matrix of input data vector.

\section{2) B. Recursive Least Square Algorithm}

The recursive least squares (RLS) is an algorithm which recursively finds the filter coefficients that minimize a weighted linear least squares cost function relating to the input signals. The recursive least-square (RLS) algorithm does not require any matrix inversion computations as the inverse correlation matrix is computed directly. The recursive least-squares (RLS) algorithm uses a different approach in carrying out the adaptation. Contrary to the LMS algorithm, which uses the steepest descent method to determine the complex weight vector, the Recursive Least Squares (RLS) algorithm uses the method of least squares. The weight vector $w(k)$, is updated by minimizing an exponentially weighted cost function that consists of the sum of error squares over a time window, in the method of steepest-descent, on the other hand, we choose the weight vector to minimize the ensemble average of the error squares. The derivation of RLS is a deterministic method while others are just stochastic.

The RLS algorithm can be described by the following equations [18]

$$
\begin{aligned}
& z(k)=\frac{\lambda^{-1} P(k-1) x(k)}{1+\lambda^{-1} x^{H}(k) P(k-1) x(k)} \\
& \alpha(k)=d(k)-w^{H}(k-1) x(k) \\
& w(k)=w(k-1)+z(k) \alpha^{*}(k) \\
& P(k)=\lambda^{-1} P(k-1)-\lambda^{-1} z(k) P(k-1)
\end{aligned}
$$

The initial value of $P(k)$ can be set to

$P(0)=\delta^{-1} I$

where $I$ is the $M X M$ identity matrix, and $\delta$ is a small positive constant for high SNR and large positive constant for low SNR. 
If compared to most of its competitors [16], RLS is a desirable algorithm because it has the ability to retain information about the input data vector, $(k)$, since the moment the algorithm was started. Therefore, the convergence of the RLS algorithm is much greater than that of the LMS algorithm by nearly an order of magnitude, but at the cost of increased computational complexity. An important feature of the RLS algorithm is its ability to replace the inversion of the covariance matrix in the Weiner solution with a simple scalar division.

\section{3) C. Sample Matrix Inversion (SMI) Algorithm}

The LMS is a continuously adaptive algorithm; one of its drawbacks is that the algorithm must go through much iteration before satisfactory convergence is achieved. If the signal characteristics are rapidly changing, the LMS adaptive algorithm may not allow tracking of the desired signal in a satisfactory manner. Therefore when the transmission is discontinuous, a block adaptive approach

The sample matrix is a time average estimate of the array correlation, matrix using K-time samples. If the random process is ergodic in the correlation, the time average estimate will be equal to the actual correlation matrix. It provides a good performance in a discontinuous traffic. However, it requires that the number of interferers and their positions remain constant during the duration of the block acquisition.

The estimates of the matrices over a block size $\mathrm{N}_{2}-\mathrm{N}_{1}$ are given by:

$$
\begin{aligned}
& R=\sum_{i=N_{1}}^{N_{2}} x(i) x^{H}(i) \\
& \hat{p}=\sum_{i=N_{1}}^{N_{2}} d^{*}(i) x^{H}(i)
\end{aligned}
$$

Where $\mathrm{N}_{1}$ and $\mathrm{N}_{2}$ form the lower and the upper limit of the observation interval, $\widehat{R}$ and $\hat{p}$ are the estimates of the covariance matrix $R$ and the cross correlation matrix $p$ respectively. The weight vector can now be estimated by the following equation:

$$
\widehat{w}=\hat{R}^{-1} \hat{p}
$$

\section{SIMULATION SETUP}

The simulations for the LMS, SMI and RLS were set in the same condition to be able to compare the performance of each algorithm in tracking and enhancing the desired users, while suppressing the interference by placing null beamforming towards the interfering signal. The setup below illustrates the simulation procedures. would give a better performance than a continuous approach. One possible approach to circumventing the relatively slow convergence of the LMS scheme is by the use of SMI method [8], [19], [20], [21], [22].

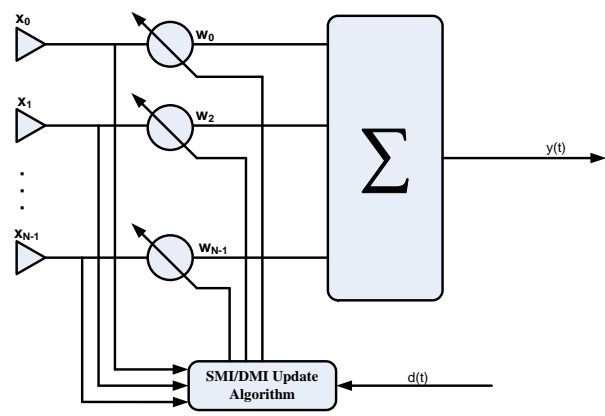

Figure 3: SMI/DMI adaptive beamforming Network

For the case of simulation 8-element linear array with 0.5 wavelength spacing was considered. The angle of arrival for the desired signal $x(t)$ is said to be $d_{1}$. At the same time the angle of arrival for the interfering signal $u(t)$ of the same form with $x(t)$ is said to be $d_{i}$ where $i=1,2,3 \ldots$ In order to simplify the simulation, the reference signal $d(t)$ was assumed to be equal to desired signal. The comparison of these algorithms is based on the depth of nulls placed towards the interferers, and the amplitude enhanced towards the desired user for each algorithm.

\section{SIMULATION RESULTS}

For tracking the desired user through steering the beam the simulation was done where only Angle of arrival of the desired user changing while the interferer remain unchanged and later both the desired and interferer were changing. The aim of this setting is to check if the given algorithm can steer the beam towards a desired user while placing the nulls towards interferers. It has been preferred that at the first the desired user changes its angle of arrival from $20^{\circ}$ to $-25^{\circ}$ while all the interferers remain unchanged. After that both the desired user and interferer changes their angle of arrival such that $d_{1}=70^{\circ}, d_{2}=-55^{\circ}$, $d_{3}=-35^{\circ}$ and $d_{4}=40^{\circ}$. The simulations results for each algorithm have been presented in figure 4-6 below.

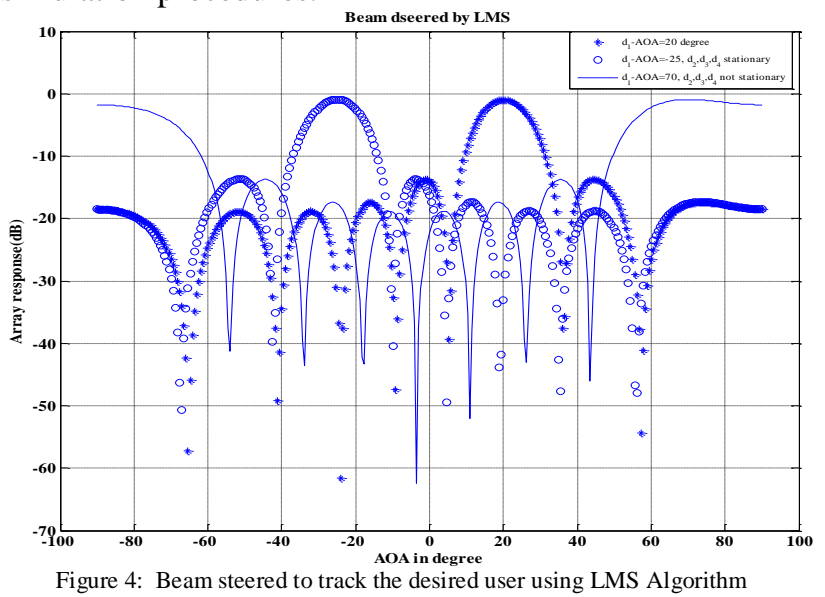




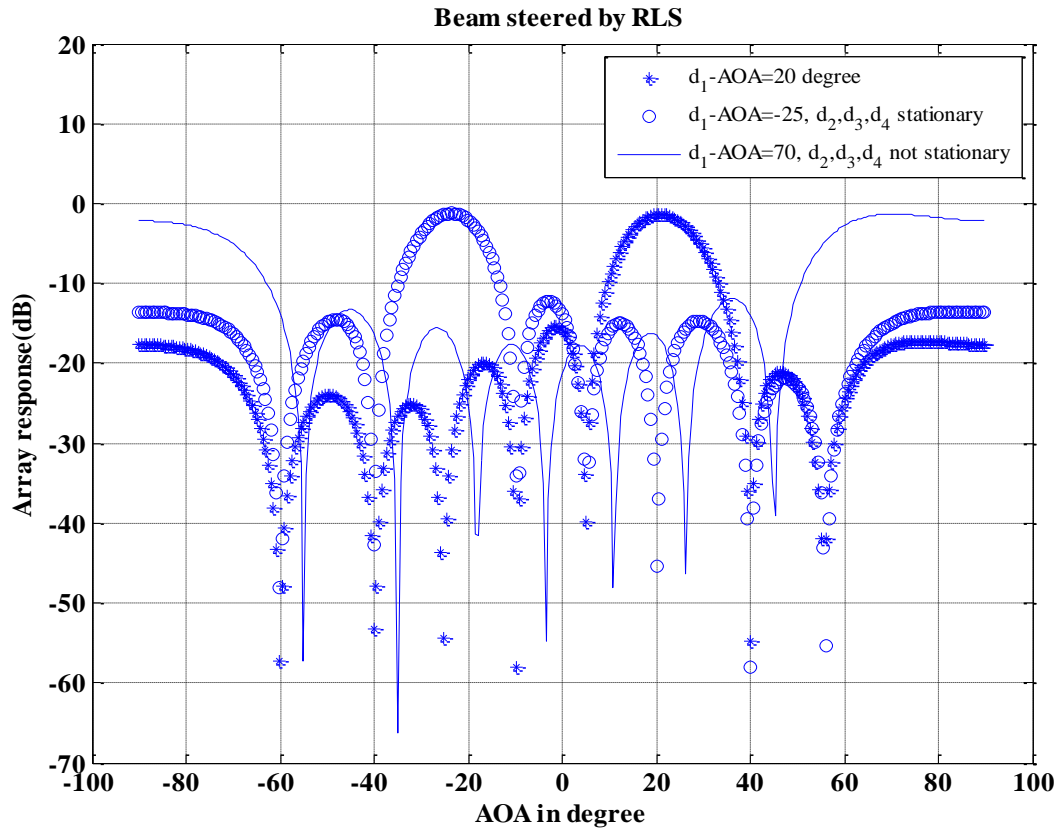

Figure 1: Beam steered to track the desired user using RLS Algorithm

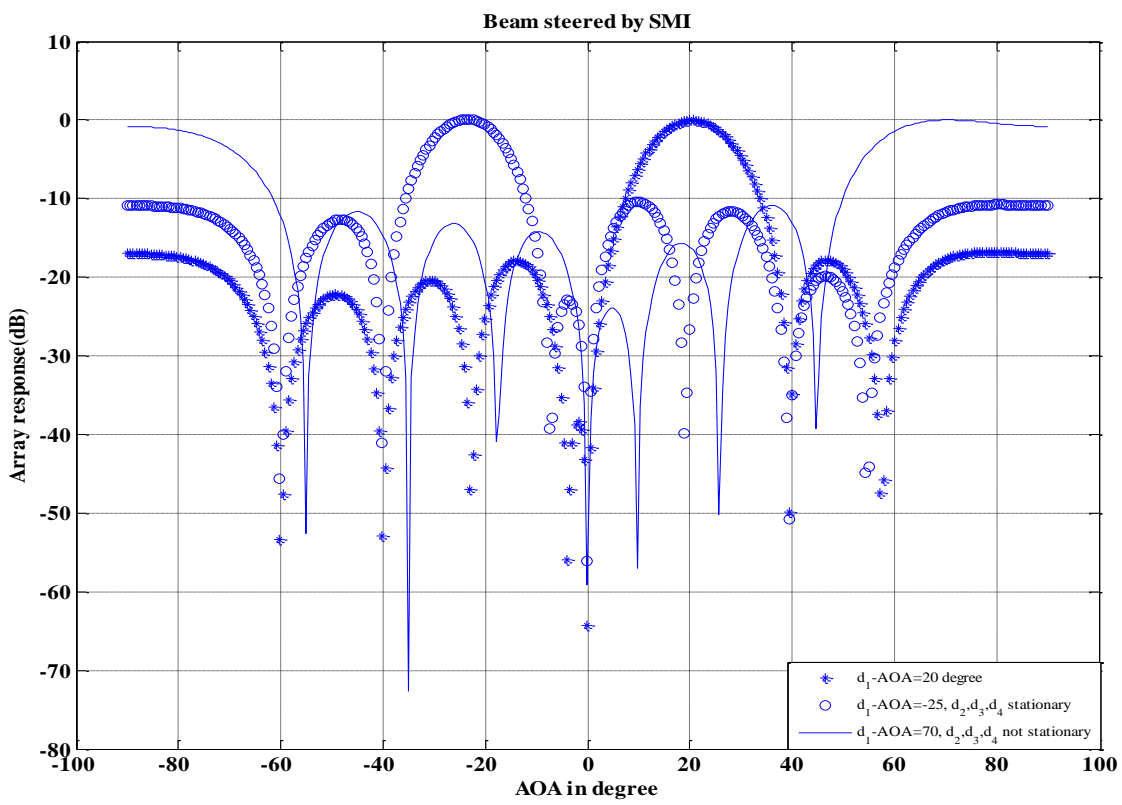

Figure 2: Beam steered to track the desired user using SMI Algorithm

One of the desired features of the wireless is even worse. In case of RLS it places the nulls to the communication is to give a reliable communication to the right interferer while providing a very deep null to the users. Since most of the users are not stationary, they keep third interferer d4. Similarly with the SMI, it can be on changing their location from time to time, thus the observed that the depth of nulls have been reduced as well. system must be able to track without disconnecting the This indicates that when the desired user changes its AOA, desired user. In this section we discuss the simulation the effectiveness of each algorithm in placing nulls results obtained when the desired user changes its AOA. It towards interferers is affected to a certain extent.

observed that all the algorithms steers their main beam When both the desired users and interferers change their towards the desired user while placing nulls to the AOA, it can be observed from simulation that all interferer. algorithms track the desired user and place the nulls to the However, when only desired user changes its AOA while interferers accordingly with the exception of LMS. The interferers are unchanged, almost all algorithms plots numerical values extracted from the simulation for all shows that the depth of the nulls have reduced to some algorithms have been summarized and presented in table 1 extent. For LMS the divergence from the interferer AOA and 2 
.Table 1: Numerical Value depicted from simulations for null depth, Amplitude enhancement and Maximum side lobes for each algorithm when desired user changes its AOA to $-25^{0}$ while interferer remain unchanged

\begin{tabular}{|c|c|c|c|c|c|}
\hline \multirow{3}{*}{ Algorithm } & Amplitude diff & \multicolumn{2}{|c|}{ Null depth performance ( $\mathrm{dB})$} & & \multirow[t]{3}{*}{ Max Side lobe } \\
\hline & $\begin{array}{l}\text { beam \& Max side } \\
\text { lobe }\end{array}$ & $\mathrm{AOA}(\mathrm{deg})$ & $\begin{array}{l}\mathrm{AOA} \\
\text { (deg) }\end{array}$ & $\mathrm{AOA}(\mathrm{deg})$ & \\
\hline & & $\mathrm{d}_{2}=-60$ & $\mathrm{~d}_{3}=-40$ & $\mathrm{~d}_{4}=40$ & \\
\hline LMS & 12.60 & -19.08 & -19.12 & -21.54 & -13.67 \\
\hline RLS & 10.91 & -46.80 & -41.41 & -56.84 & -12.21 \\
\hline SMI & 10.30 & -45.53 & -41.02 & -48.48 & -10.30 \\
\hline
\end{tabular}

Table 2: Numerical Value depicted from simulations for null depth, Amplitude enhancement and Maximum side lobes for each algorithm when both desired user and interferers changes their AOA.

\begin{tabular}{|c|c|c|c|c|c|c|}
\hline \multirow[b]{2}{*}{ Algorithm } & Amplitude diff & \multicolumn{3}{|c|}{ Null depth performance ( dB) } & \multirow{2}{*}{\multicolumn{2}{|c|}{$\begin{array}{l}\text { Max } \\
\text { lobe }\end{array}$}} \\
\hline & $\begin{array}{l}\text { beam \& Max side } \\
\text { lobe }\end{array}$ & $\begin{array}{l}\mathrm{AOA}(\mathrm{deg}) \\
\mathrm{d}_{2}=-55\end{array}$ & $\begin{array}{l}\mathrm{AOA}(\mathrm{deg}) \\
\mathrm{d}_{3}=-35\end{array}$ & $\begin{array}{l}\mathrm{AOA}(\mathrm{deg}) \\
\mathrm{d}_{4}=45\end{array}$ & & \\
\hline LMS & 12.84 & -29.01 & -31.33 & -22.00 & -13.73 & \\
\hline RLS & 10.60 & -56.02 & -65.02 & -39.00 & -11.93 & \\
\hline SMI & 10.91 & -52.56 & -73.35 & -39.41 & -10.91 & \\
\hline
\end{tabular}

\section{CONCLUSION AND FUTURE WORKS}

It has been shown that all the three algorithms direct their beams towards a desired user, and place nulls towards interferer. However the rate of their performance differs from each other while SMI gives a better performance in placing nulls towards the interference. Also LMS enhances the desired signal very well but it is poor in overcoming the interference. The RLS is closer in performance with SMI but its computational complexity is high.

In this paper we focused on non-blind adaptive beam forming algorithms therefore the future work will consider a blind adaptive algorithm. Since Cognitive Radio (CR) requires an algorithm with high convergence rate, ability to interference cancellation and tracking of desired users, in the future embedding the smart antenna with adaptive algorithms in Cognitive radio will be considered.

\section{REFERENCES}

[1] R K Jain, SumitKatiyar N K Agrawal, Smart Antenna for Cellular Mobile Communication, VSRD-IJEECE, Vol. 1 (9), 2011, 530-541.

[2] Agius A A. Leach S M. Suvannapattana P, Lund T \& Saunders S R "Intelligent Handheld Antennas for Mobile Communications Beyond the 2nd Generation" version 2.0.2, p12.

[3] Trent K, Are Smart Antennas the way to Non-Line-of-Sight? http://www.shorecliffcommunications.com/magazine/volume.asp (2001).

[4] Litwa J, Digital Beam forming in wireless communications, 1996.

[5] Steyskal H, Digital Beam forming antennas, An introduction, Microwave J, 30 (1987) 107-124.

[6] Martin Cooper, Marc Goldburg, "Intelligent Antennas: Spatial Division Multiple Access" Annual Review of Communications, 1996.

[7] J. S. Blogh and L. Hanzo. Third Generation Systems and Intelligent Wireless Networking: Smart Antennas and Adaptive Modulation. John Wiley \& Sons Inc.New York, 2002.

[8] John Litva and Titus Kwok-Yeung Lo.Digital Beamforming in Wireless Communications, Artech House, Boston, 1996.

[9] Lal, C. Godara. Applications of Antenna Arrays to mobile Communications, Part I: Performance Improvement, feasibility, and System Considerations. Proceedings of the IEEE, Vol. 85, No. 7, July 1997.

[10] Okamoto, Garret T. Smart Antenna Systems and Wireless Lans, New York Kluwer Academic Publishers, 2002.
[11] Michael Chryssomallis, "Smart antennas," IEEE Antenna and Propagation Magazine, vol. 42, pp. 129-136, 2000.

[12] SalanDurrani, Investigations into Smart Antennas for CDMA Wireless Systems, A thesis for the degree of Doctor of Philosophy The University of Queensland, Brisbane, Australia. August 2004

[14] A. Perez-Neira, X. Mestre, and J. R. Fonollosa, "Smart antennas in software radio base stations," IEEE Communications Magazine, vol. 32, no. 2, pp.166-173, February 2001.

[15] Costantine A. Balanis , Salvatore Bellofiore , Jeffry Foutz and Andreas S. Spanias, "Smart-antenna system for mobile communication networks part1:overview and antenna design," IEEE Antennas and Propagation Magazine, vol. 44, pp. 145-154, 2002.

[13] N G Chee, Desmond. Smart Antennas for Wireless Applications and Switched Beamforming, undergraduate thesis, University of Queensland, School of Information Technology and Electrical Engineering, 2001.

[16] Simon Haykin, Adaptive Filter Theory, Prentice Hall, Englewood Cliffs, New Jersey, 1996.

[17] B. Widrow, P. E. Mantey, L. J. Griffiths, and B. B. Goode, "Adaptive antenna systems," Proc. IEEE, pp. 2143\{2159, December 1967.

[18] A. J. van Veen, S. Talwar, and A. Paulraj, "A subspace approach to blind space-time signal processing for wireless communication systems", IEEE Trans. On Signal Processing, vol. 45, no.1, pp.173190, Jan. 1997.

[19] Lal, C. Godara. Applications of Antenna Arrays to mobile Communications, Part II: Beam-Forming and Direction-of-Arrival Consideration, Proceedings of the IEEE, Vol. 85, No. 8, August 1997.

[20]. Litva, J., and T. Kowk-Yeung Lo, Digital Beamforming in Wireless Communications, Artech House, 1996.

[21]. Burg, J.P., "The Relationship between Maximum Entropy Spectra and Maximum Likelihood Spectra," Geophysics, Vol. 37, pp. 375-376, April 1972.

[22]. Godara, L., Smart Antennas, CRC Press, Boca Raton, FL, 2004. 